\title{
Apego materno-fetal, ansiedade e depressão na gestação de alto risco
}

\section{Maternal-fetal attachment, anxiety and depression in high risk pregnancy}

\author{
Bruna Santana de Souza Soares ${ }^{1}$, Aline Groff Vivian ${ }^{2}$, Jussara Alves Pinheiro Sommer ${ }^{2}$
}

\section{RESUMO}

$\mathrm{O}$ apego materno fetal caracteriza-se pela intensidade com a qual a gestante manifesta sentimentos e cuidados pelo bebê. $\mathrm{O}$ estudo investigou os níveis de apego materno-fetal, ansiedade e depressão em gestantes de alto risco. Trata-se de pesquisa quantitativa, descritiva, realizada com 37 gestantes de alto risco em acompanhamento pré-natal, em Hospital Universitário da região metropolitana de Porto Alegre/RS. Foram coletadas informações da ficha sociodemográfica, Escala de Apego Materno-Fetal e dos Inventários Beck de Depressão (BDI) e Ansiedade (BAI). Os resultados indicaram (97,3\%) de apego máximo e (2,7\%) de apego médio, dentre as mulheres. Quanto à classificação do BAI e BDI, a maioria das gestantes apresentou ansiedade mínima (35,2\%) e depressão mínima (43,3\%). A depressão grave foi identificada em cinco gestantes $(13,5 \%)$ e a ansiedade grave em uma (2,7\%). O comprometimento do apego materno-fetal pode implicar em risco para depressão pós parto (DPP), especialmente em gestantes de alto risco. A presente investigação visou contribuir para a elaboração de estratégias que viabilizem a promoção da saúde materno infantil.

Palavras-chave: Apego Materno-Fetal (AMF); Ansiedade; Depressão; Gestantes

\section{ABSTRACT}

Maternal-fetal attachment is characterized by the intensity with which the pregnant woman expresses feelings and care for the baby. The study investigated the levels of maternal-fetal attachment, anxiety and depression in high-risk pregnant women. This is a quantitative, descriptive research carried out with 37 high-risk pregnant women undergoing prenatal care at a University Hospital in the metropolitan region of Porto Alegre/RS. Information was collected from the sociodemographic form, the Maternal-Fetal Attachment Scale and the Beck Depression Inventory (BDI) and Anxiety Inventory (BAI). The results indicated $(97.3 \%)$ of maximum attachment and $(2.7 \%)$ of medium attachment, among women. Regarding the BAI and BDI classification, most pregnant women had minimal anxiety (35.2\%) and minimal depression $(43.3 \%)$. Severe depression was identified in five pregnant women $(13.5 \%)$ and severe anxiety in one $(2.7 \%)$. Impaired maternal-fetal attachment may be at risk for postpartum depression (PPD), especially in high-risk pregnant women. The present investigation aimed to contribute to the elaboration of strategies that facilitate the promotion of maternal and child health.

Keywords: Maternal-Fetal Attachment (MFA); Anxiety; Depression; pregnant women

\footnotetext{
${ }^{1}$ Universidade Luterana do Brasil, ULBRA/Canoas, RS

*E-mail: lielbruna@gmail.com

${ }^{2}$ Universidade Luterana do Brasil
} 


\section{INTRODUÇÃO}

O apego materno-fetal envolve os cuidados da mãe para com o filho e inicia-se na fase gestacional (CRANLEY, 1981). Azevedo, Hirdes e Vivian (2020) apontam que o diagnóstico da gravidez de alto risco pode constituir impacto traumático à gestante, intensificando os sintomas de ansiedade e depressão materna, o que pode interferir no estabelecimento do apego materno, em virtude da propensão ao desenvolvimento de depressão pós-parto (DPP). Além das questões emocionais, cabe considerar que, em decorrência de alterações hormonais do sistema neuroendócrino, comuns do ciclo reprodutivo feminino, pode haver maior vulnerabilidade e sensibilidade às mudanças de humor (KROB et al., 2017).

Gestantes de alto risco participantes de um estudo sobre apego materno fetal, na cidade de Canoas-RS. As 33 mulheres revelaram maior sensibilidade emocional, expressando sentimentos como angústia, medo, tristeza e ansiedade, oriundos da preocupação com o desfecho da gestação, por conta da classificação de alto risco (SANTOS; VIVIAN, 2018). Estudo realizado com 247 gestantes de alto risco no Estado de Alagoas identificou a presença de ansiedade em todas as mulheres, em nível baixo 91(36,8\%), moderado, $132(53,4 \%)$, alto $24(9,7 \%)$ e risco para depressão (BARROS, 2020). Outra pesquisa desenvolvida no interior de São Paulo, com 66 gestantes de alto risco e 60 gestantes de baixo risco, constatou maior frequência de sintomas de ansiedade e depressão nas grávidas de alto risco $(36,36 \%)$ do que nas de baixo risco (SONCINI et al., 2019).

Estudos sobre o apego materno fetal, ansiedade e depressão contribuem para a valorização da saúde mental da gestante e do seu processo de aceitação gravídica (LUCENA; OTTATI; CUNHA, 2019). É relevante detectar a ansiedade e depressão no pré-natal, a fim de melhorar a qualidade da assistência à saúde da grávida, Araújo (2020). Silva (2020) afirma que a identificação da depressão na gestação é primordial para a promoção da saúde da mãe e do bebê. Conhecer os fatores que predispõem a problemas psíquicos nessas mulheres possibilita melhores intervenções pelos profissionais de saúde (LIMA et al., 2020).

Sendo assim, a presente pesquisa visou investigar a existência de associação do nível de apego materno-fetal com ansiedade e depressão em gestantes de alto risco.

\section{MÉTODO}

Trata-se de um estudo quantitativo transversal, descritivo, com dados secundários do Programa Interdisciplinar de Promoção da Saúde e Atenção à Primeira Infância (VIVIAN et al., 2018). Participaram da análise 37 gestantes de alto risco em acompanhamento pré-natal no Hospital Universitário de Canoas-RS, no período de 2018 a 2019. Todas as participantes eram maiores de 18 anos e assinaram o termo de consentimento livre e esclarecido - TCLE. 
Os instrumentos aplicados foram, Ficha de dados sociodemográficos,d, em que foi coletada a idade, local de moradia, estado civil, escolaridade e renda Escala de Apego Materno Fetal (EAMF), Escala Beck de Depressão (BDI) e Inventário Beck de Ansiedade (BAI) às gestantes que participaram do Programa Interdisciplinar de Promoção da Saúde Materno-Infantil e Atenção à Primeira Infância (VIVIAN et al., 2018). Essas informações foram organizadas em planilhas do Excel e analisadas no programa SPSS.

A Escala de Apego Materno-Fetal (CRANLEY, 1981) busca investigar o vínculo da gestante com o feto. É composta por 24 itens, com pontuação em cinco níveis (quase sempre, frequentemente, às vezes, raramente e nunca), tendo escore variante de 24 a 120 pontos. A escala foi validada para amostra brasileira com 300 gestantes (FEIJÓ, 1999).

A Escala Beck de Depressão (BDI) consiste de 21 itens, incluindo sintomas e atitudes, cuja intensidade varia de 0 a 3 . Os itens referem-se à tristeza, pessimismo, sensação de fracasso, falta de satisfação, sensação de culpa, sensação de punição, autodepreciação, auto-acusações, idéias suicidas, crises de choro, irritabilidade, retração social, indecisão, distorção da imagem corporal, inibição para o trabalho, distúrbio do sono, fadiga, perda de apetite, perda de peso, preocupação somática e diminuição de libido. Os pontos de corte são: menor que 10 ( sem depressão ou depressão mínima), de 10 a 18 (depressão, de leve a moderada), de 19 a 29 depressão, de moderada a grave), e de 30 a 63 (depressão grave).

O Inventário Beck de Ansiedade (BAI) é composto por 21 itens, com 4 opções de respostas $(0=$ absolutamente não, $1=$ levemente, $2=$ moderadamente e $3=$ gravemente $)$. $O$ escore total varia de 0 a 63 pontos definidos em 4 níveis: mínimo (0-7), leve (8-15), moderado (16-25) ou grave (26-63). A classificação dos sintomas da ansiedade proposta em: mínimo (0-10), leve (11-19), moderado (20-30) e grave (31-63). Cabe destacar que o BDI e o BAI são instrumentos validados para o Brasil, tratando-se de escalas sintomáticas, destinadas a medir a gravidade dos sintomas da ansiedade e depressão.

O presente estudo foi aprovado pelo Comitê de Ética em Pesquisa (CEP) da Universidade Luterana do Brasil, sob parecer $n^{\circ} 2.448 .176 / 2018$. As integrantes dos grupos do "Programa Interdisciplinar de Promoção da Saúde Materno-Infantil e Atenção à Primeira Infância" consentiram em participar da pesquisa com assinatura do Termo de Consentimento Livre e Esclarecido.

\section{RESULTADOS}

Participaram do estudo 37 gestantes de alto risco em acompanhamento pré-natal no Hospital Universitário de Canoas-RS, nos anos de 2018 e 2019. A figura 1 aborda o nível de apego das gestantes participantes da pesquisa. 
Figura 1 - Classificação do nível de apego das gestantes de alto risco em acompanhamento prénatal no Hospital Universitário de Canoas-RS internadas em 2018 e 2019

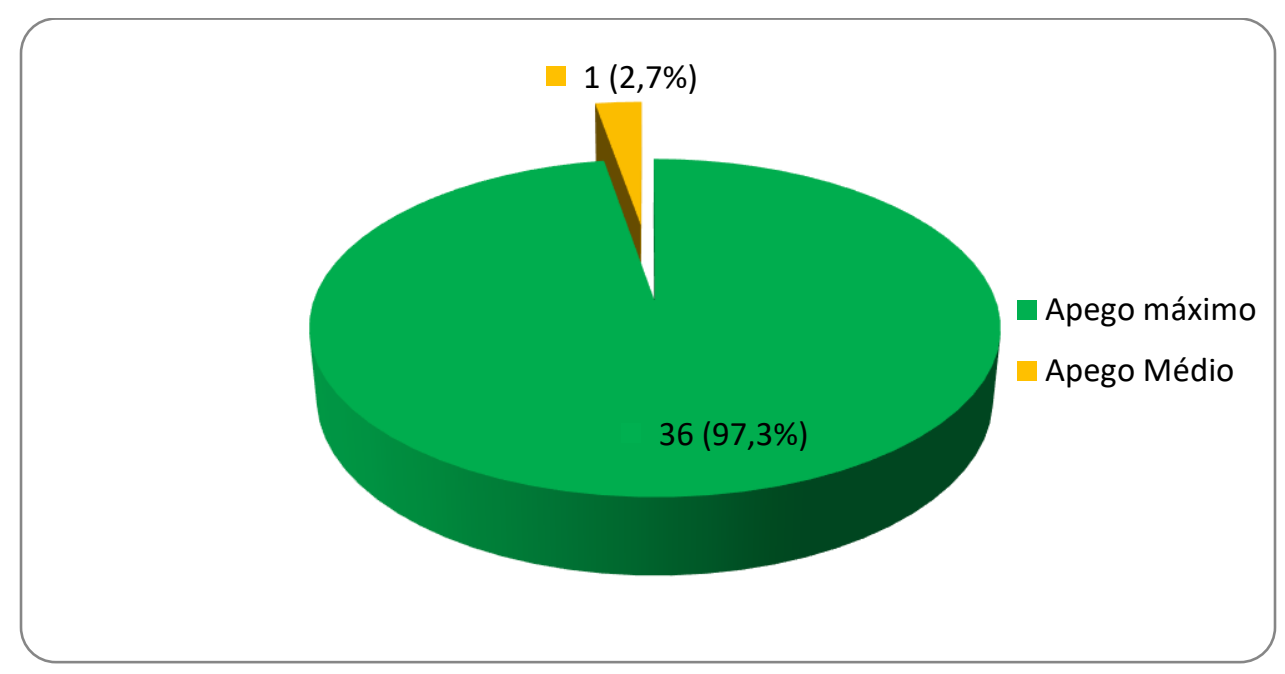

Fonte: Dados da pesquisa (2018-2019)

Dentre as gestantes investigadas, uma apresentou nível de apego médio (2,7\%), e as demais, nível de apego máximo (97,3\%). A relação afetiva da mãe com o feto, contribui para o desenvolvimento de seus circuitos cerebrais (PLUCIENNIK, 2015). Tais interações mostram-se benéficas ao desenvolvimento do neonato em relação à cognição, linguagem e comunicação (LISBOA e FERNANDES, 2021) Logo, o apego materno-fetal favorece a vida intra e pós uterina de uma criança.

Na tabela a seguir (tabela 1) observamos a classificação dos níveis de ansiedade e depressão, conforme resultado da pesquisa em destaque.

Tabela 1 - Classificação do BAI e BDI de gestantes de alto risco em acompanhamento prénatal no Hospital Universitário de Canoas-RS internadas em 2018 e 2019:

\begin{tabular}{lc}
\hline Variáveis 2 & $\mathrm{n}=37(\%)$ \\
\hline BAI: & $10(27 \%)$ \\
Leve & $13(35,2 \%)$ \\
Mínimo & $8(21,6 \%)$ \\
Moderado & $5(13,5 \%)$ \\
Grave & $1(2,7 \%)$ \\
Não aplicado & \\
BDI & $16(43,3 \%)$ \\
Mínimo &
\end{tabular}


Leve

$11(29,7 \%)$

Moderado

$5(13,5 \%)$

Grave

$1(2,7 \%)$

Incompleto/Não Aplicado

$4(10,8 \%)$

Resultados expressos através de análise de frequência 5

Fonte: Dados da pesquisa (2018-2019).

No que tange a classificação da escala do Inventário Beck de Ansiedade (BAI) e Beck de Depressão (BDI) conforme a (Tabela 1), a maioria das gestantes foram classificadas com depressão mínima $(43,3 \%)$ e $(35,2 \%)$ ansiedade mínima. A depressão grave foi identificada em uma gestante $(2,7 \%)$ e a ansiedade grave em cinco gestantes $(13,5 \%)$. No processo investigativo a escala de BAI não foi aplicada a uma paciente $(2,7 \%)$ e a de BDI em quatro (10,8\%). O estudo investigou ainda, a relação dos níveis de apego materno-fetal com o resultado das escalas BAI e BDI conforme tabela 2 a seguir.

Tabela 2 - Associação entre o apego materno com as escalas de ansiedade (BAI) e depressão (BDI).

\begin{tabular}{lccc}
\hline Escalas & \multicolumn{2}{c}{ Apego materno } & \\
$\mathrm{n}=1$ & Máximo & Valor de $\mathrm{p}$ \\
\hline BAI & $0(0 \%)$ & $5(13,9 \%)$ & 0,37 \\
Grave & $0(0 \%)$ & $10(27,8 \%)$ & \\
Leve & $0(0 \%)$ & $13(36,1 \%)$ & \\
Mínimo & $1(100 \%)$ & $7(19,4 \%)$ & 0,27 \\
Moderado & & $1(2,8 \%)$ & \\
BDI & $0(0 \%)$ & $11(30,6 \%)$ & \\
Grave & $0(0 \%)$ & $16(44,4 \%)$ & \\
Leve & $0(0 \%)$ & $4(11,1 \%)$ & \\
Mínimo & $1(100 \%)$ &
\end{tabular}

Resultados expressos através de análise de frequência

Fonte: Dados da pesquisa (2018-2019).

Teste exato de Fisher 
$\mathrm{Na}$ associação entre o nível de apego materno-fetal, ansiedade e depressão nas gestantes, identificou-se que a maioria apresentou nível de apego máximo, independentemente da escala de ansiedade e depressão (tabela 2). Não foi verificada associação estatisticamente significativa entre as escalas de apego materno com a de depressão $(\mathrm{p}=0,27)$ e ansiedade $(\mathrm{p}=0,37)$. utilizou-se o teste exato de Fischer em função das suposições do teste Qui Quadrado não serem atendidos em relação às frequências esperadas, esta análise realizou-se conforme o algoritmo de Mehta e Patel (1980), que possibilita a aplicabilidade do teste exato de Fisher para tabelas de dimensões maiores que $2 \times 2$ onde as suposições do teste 2 não forem satisfeitas. Os dados não encontrados foram considerados como missing. Não foi verificada associação estatisticamente significativa entre as escalas de apego materno com a de depressão $(p=0,27)$ e ansiedade $(p=0,37)$.

A tabela 3 abaixo, descreve os dados sociodemográficos das mulheres investigadas, e correlaciona-os com a classificação de ansiedade conforme a escala BAI.

Tabela 3 - Associação entre a classificação do BAI com as demais variáveis de estudo

\begin{tabular}{|c|c|c|c|c|c|}
\hline & \multicolumn{4}{|c|}{ BAI } & \multirow[b]{2}{*}{ Valor de $\mathrm{p}$} \\
\hline & $\begin{array}{c}\text { Mínimo } \\
\mathrm{n}=13(\%)\end{array}$ & $\begin{array}{c}\text { Leve } \\
\mathrm{n}=10(\%)\end{array}$ & $\begin{array}{c}\text { Moderado } \\
\mathrm{n}=8(\%)\end{array}$ & $\begin{array}{c}\text { Grave } \\
\mathrm{n}=5(\%)\end{array}$ & \\
\hline Idade & $30,2 \pm 6,77$ & $36 \pm 5,41$ & $34,1 \pm 6,97$ & $28,6 \pm 7,66$ & 0,18 \\
\hline Etnia & & & & & 0,26 \\
\hline Branca & $8(34,6 \%)$ & $5(27,7 \%)$ & $7(31,8 \%)$ & $2(9,1 \%)$ & \\
\hline Negra & $1(14,3 \%)$ & $2(28,6 \%)$ & $1(14,3 \%)$ & $3(42,8 \%)$ & \\
\hline Parda & $4(66,7 \%)$ & $2(33,3 \%)$ & $0(0 \%)$ & $0(0 \%)$ & \\
\hline Estado civil & & & & & 0,22 \\
\hline Casada & $6(46,1 \%)$ & $5(38,5 \%)$ & $1(7,7 \%)$ & $1(7,7 \%)$ & \\
\hline Divorciada & $1(50 \%)$ & $1(50 \%)$ & $0(0 \%)$ & $0(0 \%)$ & \\
\hline Solteira & $2(22,2 \%)$ & $0(0 \%)$ & $4(44,5 \%)$ & $3(33,3 \%)$ & \\
\hline União estável & $3(37,5 \%)$ & $1(12,5 \%)$ & $3(37,5 \%)$ & $1(12,5 \%)$ & \\
\hline Escolaridade & & & & & 0,47 \\
\hline Analfabeta & $1(100 \%)$ & $0(0 \%)$ & $0(0 \%)$ & $0(0 \%)$ & \\
\hline Ensino fundamental incompleto & $1(14,3 \%)$ & $2(28,6 \%)$ & $0(0 \%)$ & $4(57,1 \%)$ & \\
\hline Ensino fundamental completo & $4(50 \%)$ & $1(12,5 \%)$ & $0(0 \%)$ & $3(37,5 \%)$ & \\
\hline Ensino Médio incompleto & $1(20 \%)$ & $4(80 \%)$ & $0(0 \%)$ & $0(0 \%)$ & \\
\hline Ensino médio Completo & $2(25 \%)$ & $3(37,5 \%)$ & $1(12,5 \%)$ & $2(25 \%)$ & \\
\hline Superior Incompleto & $0(0 \%)$ & $1(50 \%)$ & $0(0 \%)$ & $1(50 \%)$ & \\
\hline
\end{tabular}


Superior Completo

Renda

Nenhuma

Até $1 \mathrm{SM}-1.100,00$

Até 2 SM -2.200,00

Até 3 SM $(3.300,00)$

Gestação planejada

Não

Sim

Perdas

Não

Sim

Contato com pai
$0(0 \%)$

$1(100 \%)$

$0(0 \%)$

$0(0 \%)$

$\begin{array}{lccc}4(26,7 \%) & 5(33,3 \%) & 5(33,3 \%) & 1(6,7 \%) \\ 1(16,7 \%) & 3(50 \%) & 1(16,7 \%) & 1(16,7 \%) \\ 7(53,8 \%) & 2(15,4 \%) & 2(15,4 \%) & 2(15,4 \%) \\ 1(50 \%) & 0(0 \%) & 0(0 \%) & 1(50 \%)\end{array}$

0,24

0,82

$9(37,5)$

$7(29,2)$

$4(16,7)$

$4(16,7)$

$4(33,3)$

$3(25)$

$4(33,3)$

$1(8,3)$

$0(0)$

Sim

$12(37,5)$

$8(25)$

$7(21,9)$

$5(15,6)$

Não

$1(25)$

$2(50)$

$0(0)$

Resultados expressos através de análise de frequência 3

Fonte: Dados da pesquisa (2018-2019).

Teste exato de Fisher

Quanto à idade das participantes, a associação não foi considerada significativa $(\mathrm{p}=0,22)$, observa-se que a menor média encontra-se na classificação grave $(\mathrm{p}=0,18)$. A ansiedade com classificação grave ocorreu principalmente nas gestantes autodeclaradas pretas $(42,8 \%)$. Na variável estado civil, em gestantes casadas, os percentuais mais elevados ocorrem nas classes leve $(46,1 \%)$ a moderado $(38,5 \%)$. Entre as solteiras a ansiedade mostrou-se moderada (44,5\%) e grave $(33,3 \%)$. O nível de escolaridade também não foi associado à classificação da ansiedade (p = 0,47). Identificou-se que gestantes com ensino fundamental incompleto tiveram 57,1\% de ansiedade grave e com ensino fundamental completo $(37,5 \%)$.

$\mathrm{Na}$ associação com a variável renda e ansiedade, verificou-se que nas participantes sem renda o grau de ansiedade leve e moderado são iguais (33,3\%), uma (6,3\%) apresentou nível grave. Nas gestações planejadas, a maioria dos casos de ansiedade ocorreu como mínima (42,9\%) e leve $(35,7 \%)$. A associação não foi considerada significativa $(\mathrm{p}=0,24)$.

Nos casos onde há contato da gestante com o pai do recém nascido $(37,5 \%)$, o grau de ansiedade foi mínimo. As perdas anteriores não foram associadas à classificação da ansiedade (p 
$=0,82)$. Para análise dos resultados dos dados sem resposta ou não realizados foram considerados como "missing".

A tabela 4 descreve os resultados da associação da depressão com as demais variáveis.

Tabela 4 - Associação entre a classificação do BDI com as demais variáveis de estudo

\begin{tabular}{|c|c|c|c|c|c|}
\hline \multirow[b]{2}{*}{ Variáveis } & \multicolumn{4}{|c|}{ BDI } & \multirow[b]{2}{*}{ Valor de $\mathrm{p}$} \\
\hline & Mínimo & Leve & Moderado & Grave & \\
\hline Idade: & $31,87 \pm 7,41$ & $32,54 \pm 6,94$ & $32 \pm 6,40$ & $23 \pm 0$ & 0,65 \\
\hline Etnia & & & & & 0,34 \\
\hline Branca & $10(45,5 \%)$ & $7(31,8 \%)$ & $3(13,6 \%)$ & $1(4,5 \%)$ & \\
\hline Negra & $2(28,6 \%)$ & $4(57,1 \%)$ & $1(14,3 \% \%)$ & $0(0 \%)$ & \\
\hline Parda & $4(80 \%)$ & $0(0 \%)$ & $1(20 \%)$ & $0(0 \%)$ & \\
\hline Estado Civil & & & & & 0,93 \\
\hline Casada & $7(58,3 \%)$ & $4(33,3 \%)$ & $1(8,3 \%)$ & $0(0 \%)$ & \\
\hline Divorciada & $1(100 \%)$ & $0(0 \%)$ & $0(0 \%)$ & $0(0 \%)$ & \\
\hline Solteira & $4(44,4 \%)$ & $3(33,3 \%)$ & $2(22,2 \%)$ & $0(0 \%)$ & \\
\hline União estável & $4(33,3 \%)$ & $4(33,3 \%)$ & $2(16,7 \%)$ & $1(8,3 \%)$ & \\
\hline Escolaridade & & & & & 0,91 \\
\hline Analfabeta & $1(100 \%)$ & $0(0 \%)$ & $0(0 \%)$ & $0(0 \%)$ & \\
\hline Ensino fundamental incompleto & $3(30 \%)$ & $2(20 \%)$ & $3(30 \%)$ & $1(10 \%)$ & \\
\hline Ensino fundamental completo & $2(28,6 \%)$ & $4(57,1 \%)$ & $1(14,3 \%)$ & $0(0 \%)$ & \\
\hline Ensino Médio incompleto & $4(66,7 \%)$ & $1(16,7 \%)$ & $1(16,7 \%)$ & $0(0 \%)$ & \\
\hline Ensino médio Completo & $4(57,1 \%)$ & $3(42,9 \%)$ & $0(0 \%)$ & $0(0 \%)$ & \\
\hline Superior Incompleto & $1(50 \%)$ & $1(50 \%)$ & $0(0 \%)$ & $0(0 \%)$ & \\
\hline Superior Completo & $1(100 \%)$ & $0(0 \%)$ & $0(0 \%)$ & $0(0 \%)$ & \\
\hline Renda: & & & & & 0,51 \\
\hline Nenhuma & $5(33,3 \%)$ & $4(26,7 \%)$ & $4(26,7 \%)$ & $1(6,7 \%)$ & \\
\hline Até $1 \mathrm{SM}-1100,00$ & $3(75 \%)$ & $1(25 \%)$ & $0(0 \%)$ & $0(0 \%)$ & \\
\hline Até 2 SM -2200,00 & $7(53,8 \%)$ & $5(38,5 \%)$ & $1(7,7 \%)$ & $0(0 \%)$ & \\
\hline Até 3 SM-3300,00 & $1(50 \%)$ & $1(50 \%)$ & $0(0 \%)$ & $0(0 \%)$ & \\
\hline Gestação planejada & & & & & 0,58 \\
\hline Não & $8(40 \%)$ & $8(40 \%)$ & $2(10 \%)$ & $1(5 \%)$ & \\
\hline
\end{tabular}


Sim

Contato com o pai

Sim

Não

Perdas

Não

Sim
$8(57,1 \%)$

$3(21,4 \%)$

$3(21,4 \%)$

$0(0 \%)$
$14(48,3 \%)$

$8(27,6 \%)$

$5(17,2 \%)$

$1(3,4 \%)$

$2(40 \%)$

$3(60 \%)$

$0(0 \%)$

$0(0 \%)$

0,45

0,55

$11(47,8 \%)$

$9(39,1 \%)$

$3(13 \%)$

$0(0 \%)$

$5(45,5 \%)$

(a) Resultados expressos através de média \pm desvio padrão

Demais resultados expressos através de análise de frequência

Fonte: Dados da pesquisa (2018-2019).

Teste exato de Fisher

As gestantes de alto risco classificadas com depressão grave possuem a menor média de idade, 23 anos $(\mathrm{p}=0,65)$. As autodeclaradas negras $(31,8 \%)$ manifestaram depressão leve. Todas as gestantes sem escolaridade apresentaram depressão mínima, o mesmo ocorreu com os demais níveis de escolaridade $(p=0,91)$, estando os resultados distribuídos uniformemente quanto a essa classificação, havendo a manifestação de um caso grave na escolaridade do ensino fundamental incompleto.

As gestantes sem renda foram classificadas com depressão mínima (33,3\%), leve e moderada foram (26,7\%). As participantes com gestação planejada apresentam grau de depressão mínima $(57,1 \%)$ as que não planejaram a gestação $(40 \%)$. Dentre as gestantes que não possuem contato com o pai do bebê, $60 \%$ apresentaram depressão leve. A única gestante com depressão grave possui uma perda gestacional. Para análise dos resultados, os derivados sem resposta ou não realizados foram considerados como "missing".

\section{DISCUSSÃO}

Quanto aos níveis de apego materno-fetal manifestados pelas participantes do estudo foram médio (2,7\%), e máximo (97,3\%). Resultado contrário ocorreu em estudo transversal com 280 gestantes submetidas a rastreamento de cardiopatias, com média de 27 anos $(\mathrm{DP}=7,2)$. Os níveis de apego mostraram-se predominantemente médio e alto, $74,6 \%$ e $24,6 \%$ respectivamente (ÁVILA et al. 2018). Outra pesquisa quantitativa com gestantes de baixo e alto risco apresentou dados semelhantes ao estudo em questão, em que as 25 gestantes de baixo risco gestacional manifestaram (92\%) grau máximo de apego materno-fetal, e apenas duas $(8,0 \%)$ grau médio e quanto ás 23 de alto risco, apresentaram (100\%) de apego máximo, podendo a condição de risco ter contribuído para potencialização do apego (ZEOTI \& PETEAN, 2015).

Considerando os dados da tabela 1 relacionados à classificação dos níveis de ansiedade BAI e depressão BDI, percebe-se que ambos ocorreram com maior incidência em grau mínimo, a 
classificação grave para BAI manifestou-se em cinco (13,5\%) gestantes, e para BDI em uma $(2,7 \%)$. Análise semelhante realizada com gestantes de alto risco revelou que, quanto à ansiedade, a maioria $(43,5 \%)$ apresentou nível mínimo, e duas $(8,7 \%)$, graves, e com relação à depressão, mais da metade da amostra, (56,5\%) das gestantes de risco apresentaram nível mínimo, $(26,1 \%)$, uma, grave (ZEOTI e PETEAN, 2015).

Os principais fatores de risco para ocorrência de depressão apontados foram os socioeconômicos, psíquicos, obstétricos e psicossociais (SILVA et al. 2020), tendo a depressão etiologia multifatorial. Estudo realizado no interior do Estado de São Paulo com 15 gestantes de alto risco identificou 10 fatores de risco para a depressão na gravidez, os quais foram distribuídos em socioeconômicos, psíquicos, obstétricos/maternos, e psicossociais (SILVA, 2020).

A tabela 2 que relatou a associação dos níveis de BAI e BDI com o apego materno fetal, a única gestante com apego médio, apresentou BAI e BDI moderado, concluindo-se que a condição de apego dessa mulher não sofreu potencial influência de sua saúde mental, podendo esta relacionado a outros fatores, não esclarecidos no estudo. Em pesquisa qualitativa, descritiva com caráter exploratório realizada com 33 gestantes de alto risco no Hospital Universitário de uma cidade da região metropolitana de Porto Alegre, no ano de 2018, houve dificuldade de duas gestantes em construir o apego materno-fetal devido a divergência da ex-pectativa em relação ao sexo do bebê e características individuais da gestante (SANTOS e VIVIAN, 2018).

Nas tabelas 3 e 4 verificou-se a associação entre a classificação do BAI e BDI com as variáveis sociodemográficas. Na tabela 3, apesar da etnia branca ser prevalente dentre as mulheres participantes da pesquisa, observou-se que a classificação de ansiedade grave, ocorreu principalmente nas gestantes autodeclaradas pretas $(42,8 \%)$ e , entre as solteiras, $(33,3 \%)$. Considerando a escolaridade, as gestantes acometidas com ansiedade grave tinham o ensino fundamental incompleto $(57,1 \%)$ e ensino fundamental completo $(37,5 \%)$, e a única com depressão grave informou ensino fundamental incompleto (10\%).

Estudo nacional realizado no setor de terapia intensiva neonatal em João Pessoa, afirmou a existência de adoecimento mental com ansiedade grave e depressão moderada na maioria das mães de recém-nascidos internados em unidade de terapia intensiva neonatal. Quanto à prevalência dos sintomas da ansiedade, 85 (93\%) eram graves e seis (6\%) tinham sintomas moderados. Quanto à depressão, $46(50 \%)$ possuíam sintomas moderados e $45(49 \%)$ tinham sintomas graves (SOUZA et al, 2021).

\section{CONSIDERAÇÕES FINAIS}

A presente pesquisa se propôs a investigar a associação do nível de apego materno-fetal com ansiedade e depressão, em gestantes de alto risco. A amostra estudada não evidenciou 
relação significativa entre o apego materno fetal com os níveis de ansiedade e depressão manifestados no estudo, pois as poucas gestantes que apresentaram BAI e BDI graves, expressaram apego máximo e a única com apego médio demonstrou BAI e BDI moderado.

As mulheres que apresentaram BAI grave, eram em sua maioria de etnia preta, solteiras com ensino fundamental completo, com variação de renda entre nenhuma e três salários mínimos, não planejaram a gestação, $5(22,7 \%)$, sem perdas fetais anteriores, $4(16,7 \%)$ e $15,6 \%$ relataram contato com o pai da criança.

Quanto à associação da classificação do BDI com as variáveis sociodemográficas mostraram que o único caso de depressão grave ocorreu em gestante branca, em união estável, com ensino fundamental incompleto, sem renda, gestação não planejada, com perdas anteriores e possui contato com o pai.

Os dados da amostra são insuficientes para se traçar uma análise mais conclusiva, porém sugere-se que a manifestação grave de ansiedade e depressão possa estar relacionada ao não planejamento da gravidez, pois é o único fator comum entre as seis. Estudo transversal realizado com 90 gestantes de baixo risco mostrou que 75,6 (68\%) dessas mulheres não planejaram a gravidéz e 67,6 (46\%) apresentou algum sintoma depressivo (ARAÚJO e SANTOS, 2021).

O não planejamento de uma gestação pode implicar em abalos à saúde mental feminina, como elevação dos níveis de ansiedade e risco para depressão. Silva et al., (2020), afirmam que a ansiedade é considerada uma resposta fisiológica natural do organismo, acarretando diversos sintomas emocionais, podendo estes estarem relacionados à saúde mental da gestante, pois se tornou um achado comum decorrente do ciclo de vida vivenciado pela mulher, caracterizado como momento de fragilidade emocional, flutuação hormonal e mudanças sociais que influenciam diretamente em sua saúde emocional.

O estudo pode contribuir para a implementação de estratégias de promoção da saúde materno infantil, visto que, a ansiedade e depressão em suas formas graves, ainda manifestam-se corriqueiramente entre as grávidas e possuem origem multifatorial. Sendo assim, espera-se contribuir para elaborar estratégias de promoção da saúde por equipes multidisciplinares do prénatal ao puerpério.

\section{REFERÊNCIAS}

AZEVEDO, C. C.; HIRDES, A; VIVIAN, A. G. Repercussões emocionais no contexto da gestação de alto risco. International Journal of Development Research, (IJDR), v. 10, Issue 9, pp. 40216-40220, Sep. 2020. Disponível em: https://doi.org/10.37118/ijdr.20034.09.2020. Acesso em: 3 jun. 2021. 
ARAÚJO, N. G.; SANTOS, E. S. Depressão, Ansiedade e Suporte Social na

Gestação: um estudo exploratório pré-intervenção. Revista Saúde e

Desenvolvimento Humano, 2021, Outubro, 9(3): 01-13. - ISSN 2317-8582.

http://dx.doi.org/10.18316/sdh.v9i3.7739. Acesso em Dez. 2021.

ÁVILA, C. M. et al. Rastreamento para cardiopatia: Apego materno-fetal e enfrentamento em gestantes. PSICOLOGIA, SAÚDE \& DOENÇAS, 2018, 19(2), 255 264 ISSN - 2182-8407 .Sociedade Portuguesa de Psicologia da Saúde - SPPS - www.spps.pt. Porto Alegre, RS. Disponível em: DOI: http://dx.doi.org/10.15309/18psd190208. Acesso em: 22 Dez. 2021.

BARROS, A. C. Ansiedade, traço e estado, risco para depressão e ideação suicida em gestantes de alto risco em Alagoas. 2000. 73 f. Dissertação (Mestrado) Universidade Federal de Alagoas, Maceió, PB, 2020. Disponível em: https://scholar.google.com/citations?user= Jw4qhKIAAAAJ \& hl=pt-BR . Acesso em:3 jun. 2021.

BRASIL. Ministério da Saúde. Secretaria de Atenção à Saúde. Departamento de Ações Programáticas Estratégicas. Gestação de alto risco: manual técnico. - 5. ed. - Brasília. Editora do Ministério da Saúde, 2012. 302 p. - (Série A. Normas e Manuais Técnicos).

CRANLEY, M. S. Development of a tool for measurement of maternal attachment during pregnancy. Nursing Research, v. 30, n. 5, p. 281-284, 1981. Disponível em: https://pubmed.ncbi.nlm.nih.gov/6912989/. Acesso em: 23 abr. 2021.

FEIJÓ, M. C. Validação brasileira da Maternal-fetal Attachment Scale. Arquivos Brasileiros de Psicologia, v. 51, p. 52-62, 1999. Disponível em: http://dx.doi.org/10.1590/0103-166X2015000400010. Acesso em: 19 mar. 2021.

KROB, A. D. et al . Depressão na gestação e no pós-parto e a responsividade materna nesse contexto. Revista Psicologia e Saúde, Campo Grande, v. 9, n. 3, p. 3-16, dez. 2017. Disponível em: http://pepsic.bvsalud.org/scielo.php?script=sci_arttext \& pid=S2177-093X2017000300001 \& lng= pt nrm=iso. Acesso em: 28 jun. 2021.

LISBOA, A. F.; FERNANDES, I. L. A importância do vínculo afetivo para o desenvolvimento do recém nascido: uma revisão integrativa. Revista Eletrônica Acervo Saúde | ISSN 2178-2091. REAS | Vol.13(10) . Universidade Brasil, Fernandopolis - SP. DOI: https://doi.org/10.25248/REAS.e8769.2021. Acesso em: 22 de Dez. de 2021. 
LIMA, C. M. S. COSTA, D. O.; SANTOS, J. M.; MEDEIROS, L. D.; ALVES, T. M. Perfil epidemiológico de gestantes com sinais e sintomas de depressão atendidas em uma maternidade de referência de alto risco do estado de Alagoas. 2020, 18

f.Trabalho de Conclusão de Curso - Centro Universitário Tiradentes, Maceió, AL, 2020. Disponível em:

https://openrit.grupotiradentes.com/xmlui/bitstream/handle/set/3368/TCC.pdf?sequence =1. Acesso em: 29 mai. 2021.

LUCENA, A. S; OTTATI, F; CUNHA, F. A. O apego materno-fetal nos diferentes trimestres da gestação. Revista Electrónica Internacional de la Unión Latinoamericana de Entidades de Psicología, n. 31, p. 13-24, jul. 2019. Disponível em: http://pepsic.bvsalud.org/pdf/psilat/n31/a03n31.pdf. Acesso em: 18 abr. 2021.

PLUCIENNIK, G.A. Programa Primeiríssima Infância: a experiência da atuação integrada entre as pastas de Saúde, Educação e Desenvolvimento Social. Bol. Inst. Saúde, v. 16, n. 1, 2015, p.14 -- 22.

SONCINI, N. C.; OLIVEIRA, C. M; VIVIANI, J. C; GORAYEB, R. Aspectos psicossociais em mulheres brasileiras com gestações de alto e baixo risco. Psicologia, Saúde \& Doenças, Ribeirão Preto, São Paulo, v. 20, n. 1, p. 122-136, 2019. Disponível em: http://dx.doi.org/10.15309/19psd200110. Acesso em: 13 mai. 2021.

SOUZA, L. G.; QUEIROZ, V. C.; ANDRADE, S. S. C. CÉSAR, E. S. R.; MELO, V. F. C. OLIVEIRA, S. H. S. Ansiedade e depressão em mães de recém-nascidos internos em unidade de terapia intensiva. Rev Gaúcha Enferm. 2021;42:e20200388 doi: https://doi.org/10.1590/1983-1447.2021.20200388. Acesso em: 29/12/2021

SANTOS, C. F.; VIVIAN, A. G. Apego materno-fetal no contexto da gestação de alto risco: contribuições de um grupo interdisciplinar. Diaphora, Porto Alegre, v. 7, n. 2, p. 01-10, jul/dez 2018.

DOI:https://doi.org/10.36489/nursing.2020v23i271p4961-4970. Acesso em: 25 de Dez. 2021.

SILVA, M.M.J.; LIMA, G.S.; MONTEIRO, J.C.S.; CLAPIS, M.J. Depression in pregnancy: risk factors associated with its occurrence. SMAD, Rev Eletrônica Saúde Mental Álcool Drog. 2020;16(1):1-12. doi: https://dx.doi.org/ 10.11606/issn.18066976.smad.2020.153332. Acesso em: 28 de Dez. 2021

SILVA, M.M.J; CLAPIS, M.J. Percepção das gestantes acerca dos fatores de risco para depressão na gravidez. REME - Rev Min Enferm. 2020; 24:e-1328. DOI:

10.5935/1415-2762.20200065. Acesso em: 28 de Dez. de 2021. 
SAVIANE-ZEOTE, F; PETEAN, E. B. L. Apego materno-fetal, ansiedade e depressão em gestantes com gravidez normal e de risco: estudo comparativo. Estudos de Psicologia I Campinas I 32(4) I 675-683 I outubro - dezembro 2015. https://doi.org/10.1590/0103-166X2015000400010. Acesso em :22 de Dez. de 2021

VIVIAN, A. G.; TOVO, M. F.; SALUM, T. N.; SCARPA, F. C.; PAIM, B. F. “O Bebê e Seu Mundo": Programa Interdisciplinar de Promoção da Saúde MaternoInfantil e Atenção à Primeira Infância. 2018, 9 f. Projeto de pesquisa não-publicado (Mestrado em Promoção da Saúde, Desenvolvimento Humano e Sociedade) Universidade Luterana do Brasil, ULBRA/Canoas, RS, 2018. Disponível em: DOI:10.34117/bjd 6n9-544. Acesso em: 28 mai. 2021. 